\title{
Caracterización palinológica de la miel de un Sector DE LA REGIÓN CHAQUEÑA DE LA PROVINCIA DE TUCUMÁN (ARgentina)
}

\author{
PALYNOLOGICAL CHARACTERIZATION OF HONEY FROM A SECTOR OF THE \\ Chaco Region of THE PROVINCE of Tucuman (Argentina)
}

\author{
Nora J. F. Reyes ${ }^{1 *} \mathbb{D}$, Patricia N. Asesor ${ }^{(D)}$, Verónica N. Albarracín ${ }^{3}$, María E. \\ García ${ }^{1}$ y y María L. Espeche ${ }^{1}$ (1)
}

\begin{abstract}
1. Laboratorio de Palinología, Fundación M. Lillo. Miguel Lillo 251, (T4000JFE) San Miguel de Tucumán, Tucumán, Argentina. 2. Laboratorio de Taxonomía Fanerogámica, Fundación M. Lillo. Miguel Lillo 251, (T4000JFE) San Miguel de Tucumán, Tucumán, Argentina.

3. Cátedra de Granja. Facultad de Agronomía y Zootecnia, Universidad Nacional de Tucumán, F. Ameghino s/n, Bo Mercantil, (T4105) El Manantial, Tucumán, Argentina.
\end{abstract}

*njreyes@lillo.org.ar

Citar este artículo

REYES, N. J. F., P. N. ASESOR, V. N. ALBARRACÍN, M. E. GARCÍA \& M. L. ESPECHE. 2019. Caracterización palinológica de la miel de un sector de la región chaqueña de la provincia de Tucumán (Argentina). Bol. Soc. Argent. Bot. 54: 367-379.

DOI: http://dx.doi. org/10.31055/1851.2372.v54. n3.25360

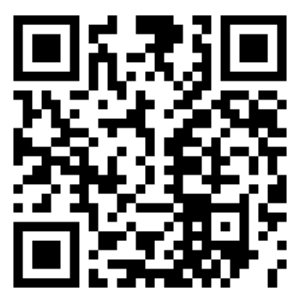

Recibido: 6 Marzo 2019 Aceptado: 22 Agosto 2019 Publicado: 30 Septiembre 2019 Editor: Gonzalo Márquez (D)

ISSN versión impresa 0373-580X ISSN versión on-line 1851-2372

\section{SUMMARY}

Background and aims: Argentina is among the leading producers and exporters of honey worldwide. Export mainly in bulk. To improve competitiveness, it launched a traceability system and quality regulations, based on the knowledge of the composition and availability of resources that allow differentiating honey by their geographical and botanical origin, data provided by the melisopalinological studies. The Chaco ecoregion and its apicultural capacity are the subject of many of these studies. In Tucumán, the Graneros Department located in this ecoregion, is promoted as a provider of beekeeping resources although the history of botanical and geographical characterization of honey is scarce. The objective of this work is the palynological characterization of honey, present in the Department Graneros of the Province of Tucumán.

M\&M: Honey samples from 3 inter-annual beekeeping campaigns were qualitatively analyzed. The present pollen morphotypes and frequency classes were determined. Data were compared by correspondence analysis.

Results: A total of 54 morphological types were identified, 14 at a specific level, 25 generic and 15 family. Of these 11 were classified as very frequent, 10 frequent, 9 infrequent and the remaining rare. $76 \%$ of the samples are monofloral, of which $46 \%$ are from Atamisquea emarginata, 31\% from Sarcomphalus mistol and $23 \%$ from Prosopis sp.

Conclusions: The interannual analysis of the honey from the Graneros Department (Tucumán), it helps to expand knowledge about the nectariferous resources of a sector of the Chaco Seco.

\section{KEY WORDS}

Honey, melisopalinology, region chaqueña, Tucumán

\section{RESUMEN}

Introducción y objetivos: Argentina se encuentra entre los principales productores y exportadores de miel a nivel mundial. Exporta principalmente a granel. Para mejorar la competitividad puso en marcha un sistema de trazabilidad y normativa de calidad, basado en el conocimiento de la composición y disponibilidad de recursos que permitan diferenciar las mieles por su origen geográfico y botánico, datos aportados por los estudios melisopalinologicos. La ecorregión del Chaco y su capacidad apícola son objeto de muchos de estos estudios. En Tucumán, el Departamento Graneros ubicado en dicha ecorregión, se potencia como proveedor de recursos apícolas a pesar de que son escasos los antecedentes de caracterización botánica y geográfica de miel. El objetivo de este trabajo es la caracterización palinológica de las mieles presentes en el Departamento Graneros de la Provincia de Tucumán.

M\&M: Se analizaron cualitativamente muestras de miel de 3 campañas apícolas interanuales. Se determinaron los morfotipos polínicos presentes y las clases de frecuencia. Los datos fueron comparados mediante un análisis de correspondencia.

Resultados: Se identificaron un total de 54 tipos morfológicos, 14 a nivel específico, 25 genérico y 15 familia. De ellos 11 fueron clasificados como muy frecuentes, 10 frecuentes, 9 poco frecuentes y los restantes raros. El $76 \%$ de las muestras son monoflorales, de ellas $46 \%$ son de Atamisquea emarginata, $31 \%$ de Sarcomphalus mistol y $23 \%$ de Prosopis sp.

Conclusiones: El análisis interanual de las mieles del Departamento Graneros (Tucumán), contribuye a ampliar el conocimiento sobre los recursos nectaríferos de un sector del Chaco Seco.

\section{Palabras clave}

Miel, melisopalinología, región chaqueña, Tucumán 


\section{INTRODUCCIÓN}

La producción y exportación de mieles en la República Argentina ha aumentado notablemente en los últimos 20 años. En la actualidad se encuentra entre los siete principales productores de miel, aportando alrededor del $4 \%$ de la producción mundial. Es el principal exportador latinoamericano a nivel mundial y junto a China son los países que poseen la mayor especialización y competitividad en el mercado mundial de la miel (Magaña-Magaña et al., 2017).

Sin embargo, a pesar de que la tendencia actual de los mercados se orienta hacia productos diferenciados por su calidad (origen botánico, origen geográfico, denominación de origen, etc.), las exportaciones argentinas se basan todavía mayoritariamente en la venta de un producto a granel. Para mejorar la competitividad de estos productos y generar un incremento en su cotización, se ha puesto en marcha un nuevo sistema de trazabilidad y norma de calidad (Ferrán et al., 2002; Ministerio de Agroindustria de la Nación, 2018). Este sistema incluye entre otras medidas el registro de los eventos vinculados al origen de la miel, como su origen geográfico y botánico. En este sentido, los estudios melisopalinológicos son los que aportan los datos de composición y disponibilidad de los recursos nectaríferos y polínicos de una región y en consecuencia permiten caracterizar las mieles (Montenegro et al., 2003; Forcone \& Andrada, 2006; Sánchez \& Vignale, 2009). Esta información imprime valor agregado a los productos melíferos (Salgado et al., 2014) haciendo posible desarrollar una denominación de origen, tal como sucede con otros productos alimenticios (Tellería, 2001). Sin embargo, es necesario que estos estudios se extiendan por varios años consecutivos para lograr una tipificación apropiada (Accorti et al., 1986) ya que existen factores, entre ellos los meteorológicos (temperatura y lluvia) y antrópicos (cultivo, forestación, desmonte, etc.), que inciden tanto en el comportamiento de las abejas como en la oferta florística modificando o condicionando los espectros polínicos (Jean-Prost, 1989; Philippe, 1990; Jato et al., 1994).

Los estudios realizados hasta el momento sobre mieles argentinas permitieron caracterizar las mieles de las diferentes ecorregiónes (Burkart et al., 1999), como las del Espinal (Lusardi et al.,
2005; Caccavari \& Fagúndez, 2010; Costa et al., 2013, 2016; Fagúndez et al., 2016), de la Pampa (Tellería, 1992, 1995, 1996; Forcone \& Tellería, 1998, 2000; Forcone et al., 2003, 2005; Naab \& Tamame, 2007) y de la Selva de Yungas y Puna (Quiroga et al., 2008; Sánchez \& Vignale, 2009; Sánchez \& Lupo, 2009, 2011, 2016; Burgos \& Sánchez, 2014; Burgos et al., 2015; Flores et al., 2015; Méndez et al., 2016). La gran extensión de la ecorregión del Chaco (Salgado et al., 2014) y su potencial apícola fueron objeto de diversos estudios melisopalinologicos en diferentes provincias (Nuñez Camelino, 2000; Basilio \& Noetinger, 2002; Montenegro \& Chifa, 2004; Cabrera, 2006; Cabrera et al., 2013; Salgado et al., 2014; Aquino et al., 2015; Carrizo et al., 2015; Salgado, 2016). Sin embargo en Tucumán son escasos los antecedentes de caracterización botánica y geográfica de miel en todos sus ambientes (Pailhe \& Popolizio, 1971; Popolizio \& Pailhé, 1973; Albarracin et al., 2014) a pesar de que posee áreas aptas para el desarrollo de la apicultura y un clima que posibilita el desarrollo de una abundante flora autóctona que propicia la producción de mieles con rasgos distintivos, como es el caso de "la miel de monte" (Albarracín et al., 2014).

El departamento Graneros, ubicado al sur de la provincia de Tucumán, en la ecorregión Chaco Seco, constituye un área, donde la actividad apícola se potencia como proveedora de recursos económicos. El objetivo de este estudio fue la caracterización palinológica de las mieles de dicho departamento como aporte al conocimiento de la flora melífera que contribuye a la producción apícola de la zona.

\section{Materiales y Métodos}

Se analizaron 34 muestras de miel de Apis mellifera L. producidas por colmenas ubicadas en cuatro localidades (Lamadrid, Viltrán, Taco Ralo y Páez) del departamento Graneros, ubicado al sureste de la provincia de Tucumán (Fig. 1).

Este departamento, se sitúa en las regiones agroecológicas Llanura deprimida y Llanura chaco pampeana (Zuccardi \& Fadda, 1985). Su vegetación natural consiste en bosques mesofíticos o xerófilos caducifolios, pertenecientes a la ecorregión Chaco Seco. En ella el clima es semiárido cálido (Zuccardi \& 

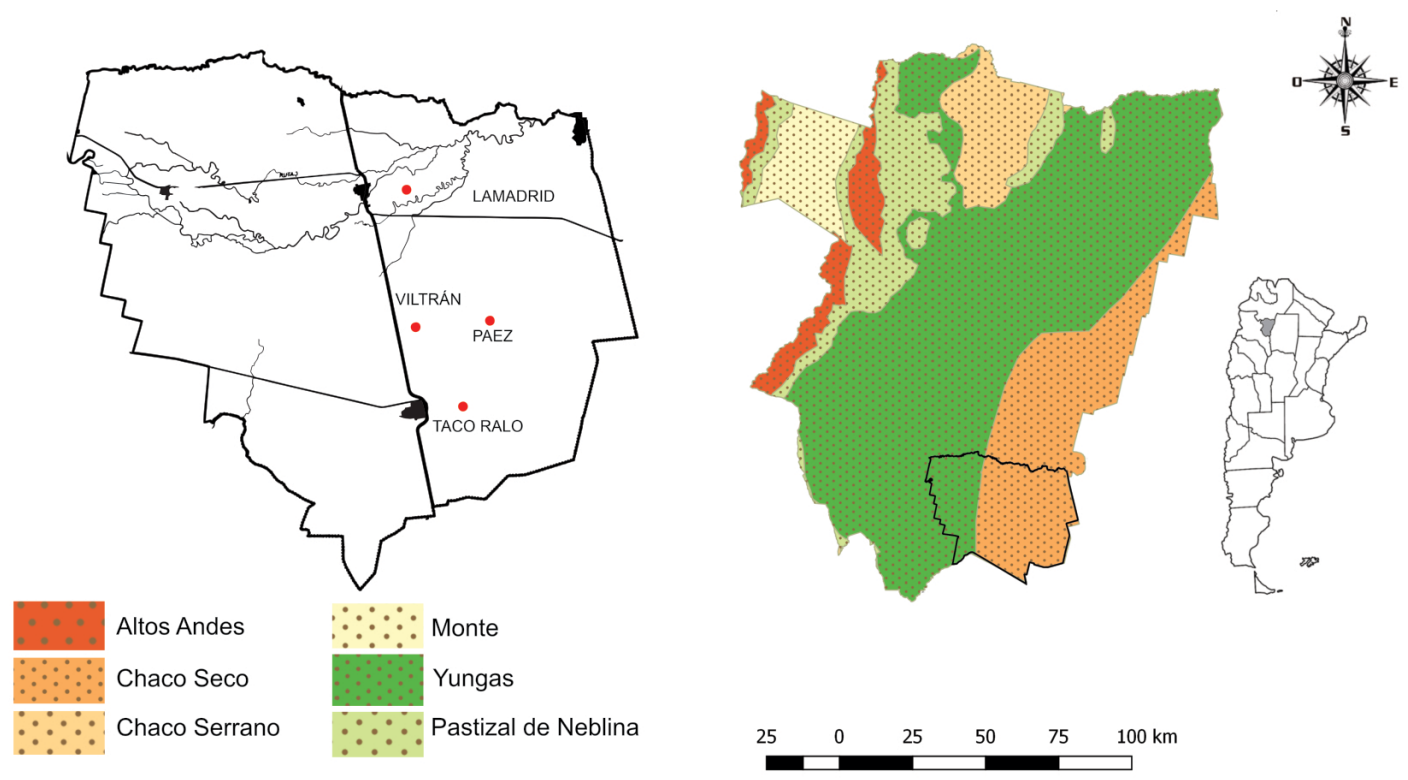

Fig. 1. Distribución geográfica de las colmenas muestreadas y ecorregiones de la provincia de Tucumán. Fuente: Dirección de Tecnologías de la información, Secretaría de Gestión Pública y Planeamientos, Gobierno de Tucumán, 2017. Infraestructura de Datos Espaciales, Provincia de Tucumán, departamento SIG. Secretaría de Planeamiento, 2010.

Fadda, 1985), con precipitaciones anuales entre 500 a $650 \mathrm{~mm}$. La mayoría de los suelos son de naturaleza salino-sódica lo que define una limitación edáfica, salinidad y alcalinidad sódica (Torrella \& Adámoli, 2006). Según el informe técnico de la Comisión de emergencia para el tratamiento de la problemática de inundaciones en el sur de la provincia de Tucumán, este de Catamarca y Río Hondo (DRH, 2017), en el departamento Graneros, los desmontes se estiman en 95.000 ha en las últimas 3 décadas. Lo que, junto con cambios en el uso del suelo como los malos manejos del ganado, la extracción forestal o la agricultura han tenido como consecuencia un aumento en el riesgo de inundaciones.

El sitio de muestreo corresponde a una zona baja, con planicies cultivadas, restos de bosques chaqueños y zonas anegadizas y salitrosas (Gonzáles et al., 2009). La vegetación está formada por pequeños bosques casi puros de algarrobos, con especies acompañantes como Aspidosperma quebrachoblanco Schltdl., Parkinsonia praecox (Ruiz \& Pav. ex Hook.) Hawkins, Sarcomphalus mistol (Griseb.) Hauenschildy, Vachellia aroma (Gillies ex Hook. \& Arn.) Seigler \& Ebinger y Senegalia praecox
(Griseb.) Seigler \& Ebinger. En el sotobosque se diferencian especies de arbustos y gramíneas entre los que se destacan Atamisquea emarginata Miers ex Hook. \& Arn., Opuntia Mill. y otros cactus.

Las muestras de mieles fueron aportadas por apicultores del Programa Cambio Rural, del Ministerio de Agroindustria de la Nación, con apoyo técnico del INTA. La oferta de floración, en la ecorregión del Chaco, a diferencia de otras regiones argentinas, se produce en dos momentos del año fines de invierno-comienzos de primavera y durante el verano (Salgado et al., 2014). Motivo por el cual algunos de los productores apícolas de las cuatro localidades realizaron estas dos cosechas anuales, resultando en tres campañas correspondientes a los períodos 2012-2013 (campaña I), 2013-2014 (campaña II) y 2014-2015 (campaña III). Las cuatro localidades donde se ubicaron las colmenas no contaron con dos cosechas en todos los casos, por lo que se unificaron los datos en cada campaña (Tabla 1). En la campaña III no se consideraron muestras de los primeros meses de 2015 (enero-marzo), debido a que las colmenas se vieron afectadas por inundación de los sectores donde estaban 
Bol. Soc. Argent. Bot. 54 (3) 2019

Tabla 1. Datos de cosechas por campaña. Abreviaturas= I: campaña 2012-2013; II: campaña 2013-2014; III: campaña 2014-2015.

\begin{tabular}{|c|c|c|c|c|}
\hline $\begin{array}{c}\mathbf{N}^{\circ} \text { de } \\
\text { Muestra }\end{array}$ & Campaña & Fecha Cosecha & Apicultor & Localidad \\
\hline 1 & I & set-12 & Juan Aparicio & Paez \\
\hline 2 & I & Oct-12 & Juan Aparicio & Paez \\
\hline 3 & 1 & Oct-12 & Miguel Scaglioni & Paez \\
\hline 4 & I & Oct-12 & Martín Ruiz & Viltrán \\
\hline 5 & I & Nov-12 & Bruno José & Taco Ralo \\
\hline 6 & I & Nov-12 & Scaglioni-Corvalán & Taco Ralo \\
\hline 7 & I & Nov-12 & Miguel Scaglioni & Taco Ralo \\
\hline 8 & I & Nov-12 & Jorge Rivalta & Taco Ralo \\
\hline 9 & I & Nov-12 & Scaglioni-Corvalán & Taco Ralo \\
\hline 10 & I & Nov-12 & Jorge Rivalta & Taco Ralo \\
\hline 11 & I & Nov-12 & Jorge Rivalta & Taco Ralo \\
\hline 12 & I & Jan-13 & Juan Aparicio & Paez \\
\hline 13 & I & Jan-13 & Gino Cantela & Lamadrid \\
\hline 14 & I & Jan-13 & Juan Aparicio & Paez \\
\hline 15 & I & Jan-13 & Juan Aparicio & Paez \\
\hline 16 & I & Jan-13 & Juan Aparicio & Paez \\
\hline 17 & I & Feb-13 & Juan Aparicio & Paez \\
\hline 18 & II & Oct-13 & Juan Aparicio & Taco Ralo \\
\hline 19 & II & Oct-13 & Martín Ruiz & Viltrán \\
\hline 20 & II & Nov-13 & Martín Ruiz & Viltrán \\
\hline 21 & II & Nov-13 & Juan Aparicio & Taco Ralo \\
\hline 22 & II & Nov-13 & Juan Aparicio & Taco Ralo \\
\hline 23 & II & Dec-13 & Juan Aparicio & Taco Ralo \\
\hline 24 & II & Dec-13 & Juan Aparicio & Taco Ralo \\
\hline 25 & II & Feb-14 & Gino Cantela & Lamadrid \\
\hline 26 & III & Aug-14 & Juan Aparicio & Taco Ralo \\
\hline 27 & III & set-2014 & Miguel Scaglioni & Taco Ralo \\
\hline 28 & III & set-2014 & Miguel Scaglioni & Taco Ralo \\
\hline 29 & III & Nov-14 & Juan Aparicio & Taco Ralo \\
\hline 30 & III & Nov-14 & Juan Aparicio & Taco Ralo \\
\hline 31 & III & Nov-14 & Juan Aparicio & Taco Ralo \\
\hline 32 & III & Nov-14 & Juan Aparicio & Taco Ralo \\
\hline 33 & III & Dec-14 & Juan Aparicio & Taco Ralo \\
\hline 34 & III & Dec-14 & Juan Aparicio & Taco Ralo \\
\hline
\end{tabular}




\section{N. J. F. Reyes et al. - Caracterización de mieles del Chaco tucumano}

emplazadas (Portal Apícola, 2015); por lo que se asumen como representativas de esta campaña, las muestras recolectadas durante los últimos meses de 2014 (noviembre-diciembre).

La economía familiar de los productores apícolas que aportaron las muestras estudiadas actualmente está representada por una ganadería de cría de ganado mayor y menor. Se localizan algunos cultivos de maíz y zapallos. En los últimos años, en suelos no salinos, se ha extendido el cultivo de soja y sorgo. Los excedentes de las producciones de autoconsumo se comercializan en ferias locales. Entre estas actividades se encuentra la producción apícola. El número de cajones por productor es aproximadamente de 70 colmenas con un manejo muy artesanal de sus apiarios, llegando, por la marginalidad de la zona a hacer un manejo casi libre de pesticidas para el control de enfermedades apícolas.

\section{Análisis de mieles}

Para el procesamiento de las muestras de miel se siguieron las normas del análisis cualitativo de Louveaux et al. (1978).

La determinación de los morfotipos polínicos se efectuó mediante comparación con colecciones de referencia. Una de estas colecciones se elaboró a partir de granos de polen proveniente de la vegetación ubicada en un área de $50 \mathrm{~m}$ de radio alrededor de los apiarios, recolectados durante la primera temporada apícola analizada. Además, se consultó la PAL-TUC (García et al., 2017) y se utilizó bibliografía especializada (Markgraf \& D’Antoni, 1978; Sáenz de Rivas, 1978; Pire et al., 1998, 2001, 2006; Hesse et al., 2009; García et al., 2012). Los tipos polínicos se determinaron a nivel de especie, género y familia. El tipo morfológico políada corresponde a las especies Senegalia gilliesii (Steud.) Seigler \& Ebinger y S. praecox (Griseb.) Seigler \& Ebinger. Dentro del morfotipo Asteracea se incluyen Baccharis sp., Eupatorium sp., Parthenium sp., Ambrosia sp. y Solidago sp.

Las observaciones y los registros fotográficos se efectuaron con Microscopio óptico Zeiss Axio LAB. A1 con cámara incorporada Axiocam ERc5s.

Para determinar clases de frecuencia de los morfotipos polínicos se siguió Louveaux et al. (1978) y Fagúndez \& Caccavari (2003). Se describieron como: polen dominante (D) más de $45 \%$, polen secundario (S) entre 15 y $45 \%$, polen de menor importancia (M) entre 3 y $15 \%$, polen en traza o raro (T) menos del $3 \%$ y polen esporádico o presente $(+)$ $<1 \%$. Los autores utilizan los términos esporádicos, raros, frecuentes y muy frecuentes para expresar la frecuencia de los granos de polen de plantas anemófilas y otras plantas con menos néctar.

Para clasificar la miel según su origen botánico se siguieron las categorías establecidas por la Resolución SAGPyA No $1051 / 94$ y su modificatoria $274 / 95$. Se consideran mieles monoflorales o uniflorales aquellas en cuya composición se encuentre, como mínimo, un cuarenta y cinco por ciento $(45 \%)$ de polen de la misma familia, género o especie floral y mieles multiflorales a las que presentan en su composición polen de varias especies vegetales, sin que ninguna de ellas pueda considerarse predominante.

\section{Análisis estadístico}

Con la finalidad de observar la relación entre los tipos polínicos que componen las mieles y los periodos de producción se aplicó un Análisis de Correspondencia, para ello se utilizó el software "Infostat versión 2011" (Di Rienzo et al., 2012). En este análisis, se tuvieron en cuenta los principales morfotipos polínicos presentes en las muestras de mieles, considerando como tales, a los presentes en tres o más cosechas. Este análisis es una técnica de ordenación que permite representar en un diagrama bi o tridimensional la asociación entre las variables analizadas en nuestro caso correspondiente a las estaciones de cada campaña, donde la proximidad entre los puntos representados (morfotipos polínicos) está relacionada con el nivel de asociación. Dentro de este análisis, los valores de porcentaje de inercia corresponden a la información contenida en cada dimensión, es decir, representan la importancia de cada una de ellas, cuanto mayor es su valor mejor representa las similitudes y diferencias entre las variables. El porcentaje acumulado y los valores Chi-cuadrado muestran a su vez la existencia de una relación significativa entre las variables.

\section{Resultados}

El análisis polínico fue llevado a cabo sobre muestras de miel de tres campañas interanuales entre los años 2012-2015. En la campaña 20122013 (I) se reconocieron 41 tipos polínicos, 33 en la campaña 2013-2014 (II) y 32 en la de 2014-2015 
(III). En total, considerando las tres campañas, se identificaron 54 tipos morfológicos, 14 de los cuales se determinaron a nivel de especie, 25 a nivel de género y 15 a nivel de familia (Tabla 2). Las clases de frecuencia de los tipos polínicos (dominantes, secundarios, de menor importancia y traza) se representan en la Fig. 2. No se incluyen en la gráfica los taxones representados por debajo del $1 \%$. Atamisquea emarginata, Prosopis sp. y Sarcomphalus mistol aparecen en las cuatro clases de frecuencia, mayormente como polen dominante.
El $76 \%$ de las muestras son monoflorales. De ellas el $46 \%$ son de $A$. emarginata, $31 \%$ de $S$. mistol y $23 \%$ de Prosopis sp. Estos tipos polínicos también aparecen en las mieles multiflorales como tipos polínicos secundarios en porcentajes que varían del 15 al $40 \%$. Otros tipos polínicos secundarios que componen las mieles multiflorales son: P. praecox, Heliotropium sp., Asteraceae, Myrtaceae, Schinus sp., Cereus sp., Celtis sp. y Solanum argentinum Bitter \& Lillo en orden decreciente (Fig.2). Cabe aclarar que Celtis sp. no se incluye en el gráfico debido a que Tabla 2. Composición de mieles monoflorales y multiflorales. Abreviaturas= I: campaña
2012-2013; II: campaña III: 2013-2014; C: campaña 3: 2014-2015.

\begin{tabular}{|c|c|c|c|}
\hline Campaña & Tipo de Miel & Cantidad & Tipos Polínicos \\
\hline 1 & Monofloral & 3 & Prosopis sp. \\
\hline I & Monofloral & 6 & Sarcomphalus mistol \\
\hline 1 & Monofloral & 6 & Atamisquea emarginata \\
\hline 1 & Multifloral & 1 & $\begin{array}{c}\text { Atamisquea emarginata } \\
\text { Sarcomphalus mistol } \\
\text { Prosopis sp. } \\
\text { Celtis sp. }\end{array}$ \\
\hline 1 & Multifloral & 1 & $\begin{array}{l}\text { Sarcomphalus mistol } \\
\text { Schinus sp. } \\
\text { Prosopis sp. }\end{array}$ \\
\hline II & Monofloral & 4 & Atamisquea emarginata \\
\hline II & Monofloral & 3 & Prosopis sp. \\
\hline II & Multifloral & 1 & $\begin{array}{l}\text { Prosopis sp. } \\
\text { Asteraceae }\end{array}$ \\
\hline III & Monofloral & 2 & Prosopis sp. \\
\hline III & Monofloral & 2 & Atamisquea emarginata \\
\hline III & Multifloral & 1 & $\begin{array}{c}\text { Atamisquea emarginata } \\
\text { Prosopis sp. } \\
\text { Parkinsonia praecox }\end{array}$ \\
\hline III & Multifloral & 1 & $\begin{array}{c}\text { Atamisquea emarginata } \\
\text { Cereus sp. } \\
\text { Heliotropium sp. }\end{array}$ \\
\hline III & Multifloral & 1 & $\begin{array}{c}\text { Atamisquea emarginata } \\
\text { Cereus sp. } \\
\text { Heliotropium sp. }\end{array}$ \\
\hline III & Multifloral & 1 & $\begin{array}{l}\text { Prosopis sp. } \\
\text { Parkinsonia praecox }\end{array}$ \\
\hline III & Multifloral & 1 & $\begin{array}{l}\text { Schinus sp. } \\
\text { Myrtaceae } \\
\text { Asteraceae } \\
\text { morfotipo } 1\end{array}$ \\
\hline
\end{tabular}




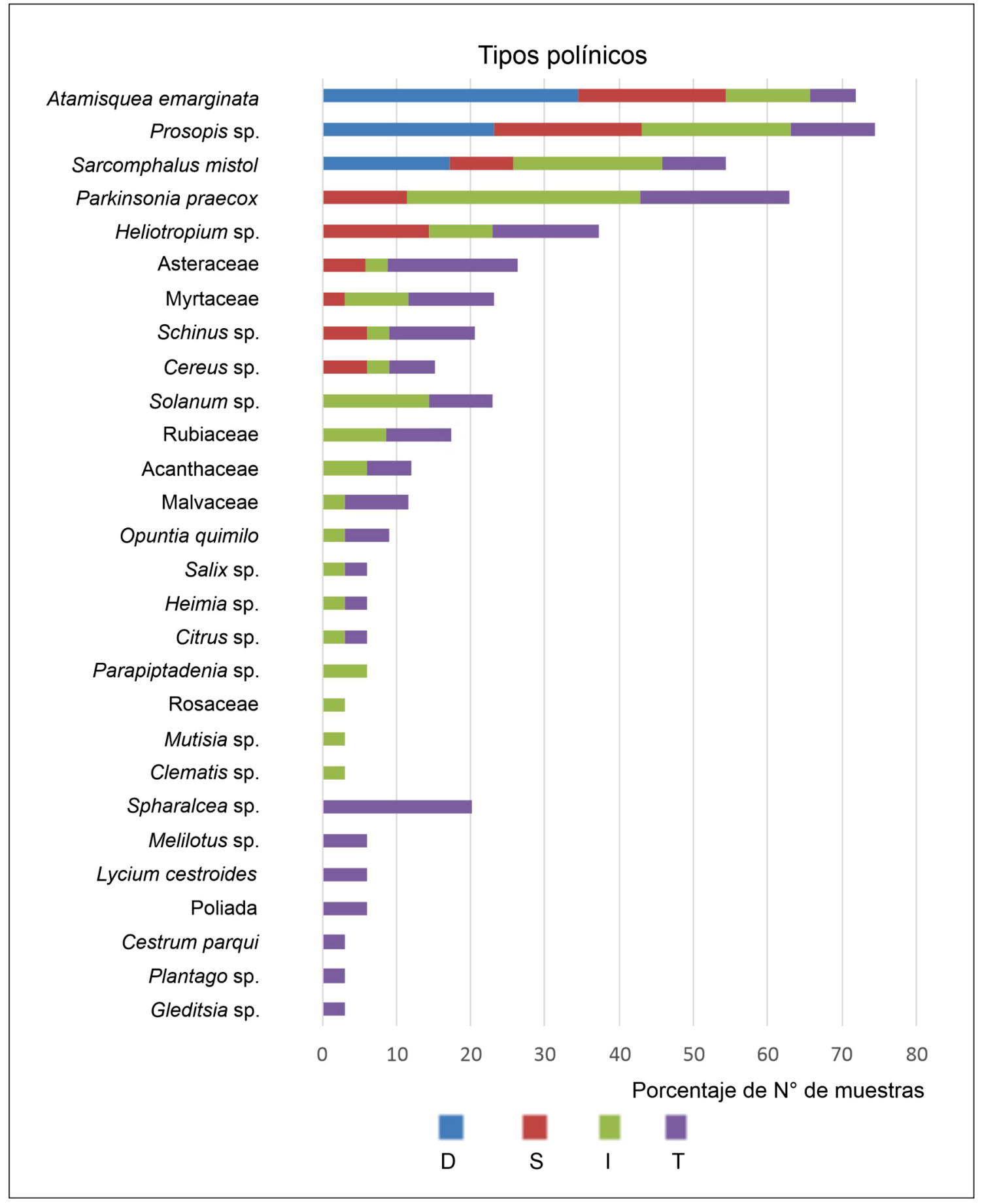

Fig. 2. Clases de Frecuencia. D: polen dominante (>45\%); S: polen secundario (16\%-45 \%); I: polen de menor importancia $3 \%-15 \%)$; T: polen traza (1\%-3\%). No se incluyen en la gráfica los taxones representados por debajo del $1 \%$. 
pertenece a la categoría de especies anemófilas. En las muestras analizadas Celtis sp. ha sido identificado en el 48,6 \% como polen esporádico, en el $23 \%$ como raro y en el $3 \%$ como frecuente. Lo mismo ocurre con Solanum argentinum, que no ostenta nectarios, pero se observó en 8,6\% de las muestras como esporádico y en un $3 \%$ como polen frecuente.

Los tipos polínicos encontrados por debajo del 1\% son: Anadenanthera colubrina (Vell.) Brenan, Allophylus edulis (A. St.-Hil., A. Juss. \& Cambess.) Hieron. ex Niederl., Chenopodiaceae, Solanum elaeagnifolium Cav., Cactaceae, Ambrosia sp., V. aroma, Urticaceae, Gomphrena sp., Poaceae, Mimosa sp., Lamiaceae, Aspidosperma quebracho-blanco Schltdl., Pinaceae, Morus sp., Juglans australis Griseb., Verbenaceae, Cyperaceae y Meliaceae. La presencia de Ambrosia sp., Chenopodiaceae, Urticaceae, Poaceae, Pinaceae, Morus sp. y Cyperceae, principalmente anemófilas, debe caracterizarse como esporádica, y se explica de la misma forma que la presencia de Celtis sp. y Solanum argentinum.
El Análisis de Correspondencia, permitió ordenar y visualizar las asociaciones entre las cosechas de cada campaña y los principales tipos polínicos, observándose en el diagrama cartesiano que la campaña II con sus dos cosechas y la primavera de la campaña III son más próximas, es decir que poseen perfiles parecidos en cuanto a la composición de tipos polínicos. Dos de los tipos polínicos más abundantes como son Atamisquea emarginata y Prosopis sp. se relacionan con estas cosechas. La primavera y el verano de la campaña I se alejan del grupo anterior relacionándose más cercanamente con otros tipos polínicos como $S$. mistol que se destaca como tipo polínico abundante y por conformar mieles monoflorales. Por último, el verano de la campaña III evidencia en el gráfico su particularidad al no contar con muestras de los meses de pleno verano. El primer eje, del diagrama explicó el 49,48 \% de la variación y el segundo el 33,06 \% (Fig. 3). La Tabla 3, muestra los resultados de los valores de porcentaje de inercia, porcentaje acumulado y Chi-cuadrado.

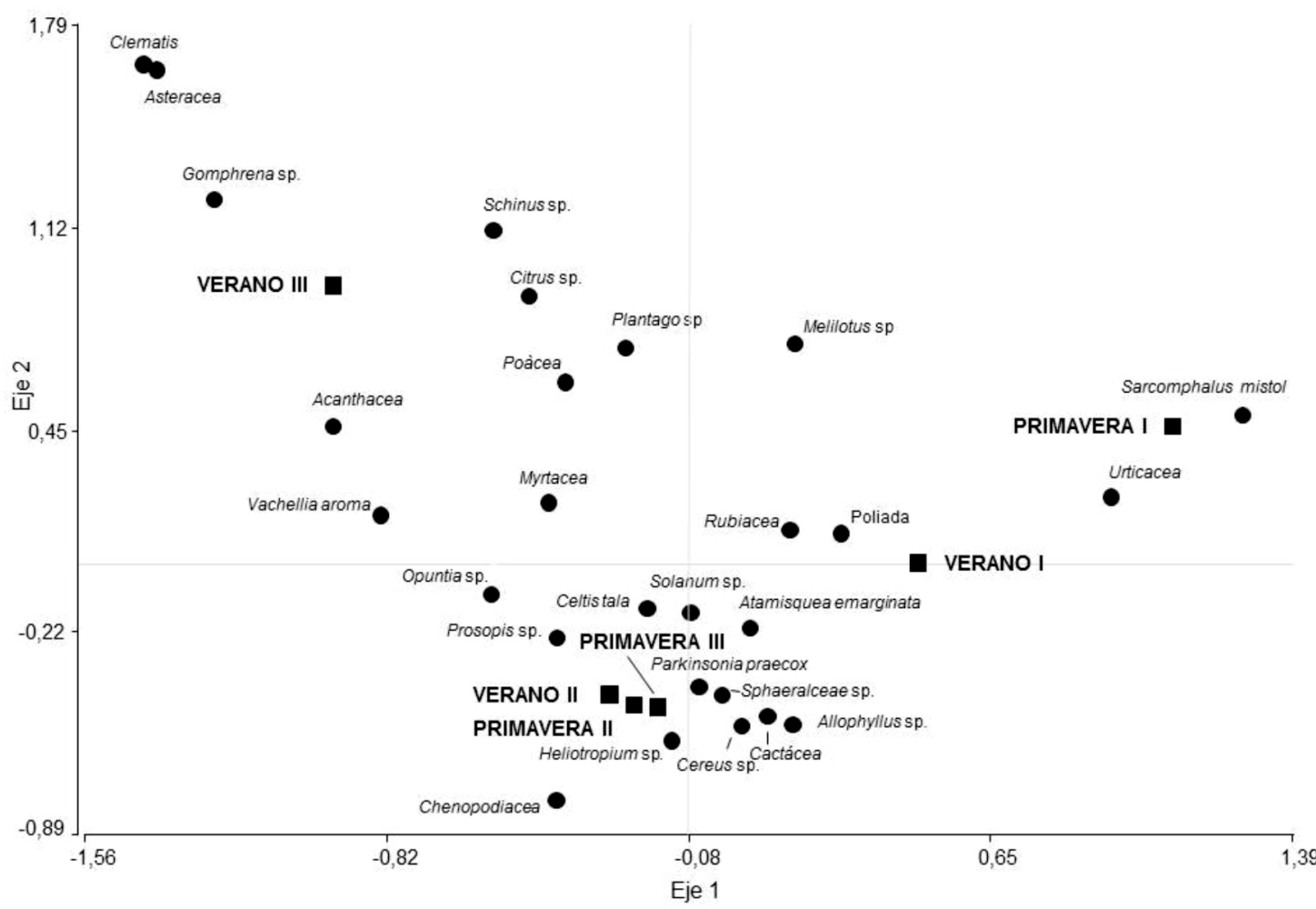

Fig. 3. Diagrama de correspondencia para los principales morfotipos polínicos y su relación con las campañas. 


\section{N. J. F. Reyes et al. - Caracterización de mieles del Chaco tucumano}

Tabla 3. Resultados de los valores de porcentaje de inercia, porcentaje acumulado y Chi-cuadrado.

\begin{tabular}{|cccccc|}
\hline & Autovalor & Inercias & Chi-Cuadrado & (\%) & \% Acumulado \\
\hline 1 & 0.64 & 0.41 & 237.16 & 49.48 & 49.48 \\
2 & 0.52 & 0.27 & 158.49 & 33.06 & 82.54 \\
\hline
\end{tabular}

\section{Discusión}

Las mieles del departamento Graneros analizadas presentan un predominio de mieles monoflorales sobre las multiflorales. Los tipos polínicos dominantes son A. emarginata, Prosopis sp. y $S$. mistol, especies típicas de la ecorregión chaqueña según Morello et al. (2012) y calificadas como plantas melíferas de primer orden para dicho ambiente por Arenas (2003). A su vez, los mismos tipos polínicos también aparecen en la conformación de mieles multiflorales como secundarios. Prosopis sp. y S. mistol cuentan con antecedentes en la producción de mieles monoflorales (Cabrera, 2006; Salgado, 2006), mientras que A. emarginata lo hace en la conformación de mieles mixtas (Salgado et al., 2014). Si bien Prosopis y Acacia han sido mencionadas como especies distintivas en la composición de mieles chaqueñas, estas también lo son en mieles de otras regiones como la Pampeana (Naab \& Tamame, 2007) y el Monte (Forcone \& Tellería, 2000).

La flora nativa, especialmente las especies arbóreas son los principales recursos para el desarrollo de las colmenas de la ecorregión chaqueña (Cabrera, 2006; Carrizo et al., 2015) lo mismo ocurre en el departamento Graneros, al observarse la composición de los tipos polínicos más abundantes. La presencia de tipos polínicos de especies anemófilas podría deberse a contaminación del néctar dentro de las colmenas por polen que las abejas llevan en sus corbículas (Soderstron \& Calderon 1971; Basilio \& Romero 1996; Salgado \& Pire 1999).

Las familias con mayor representatividad, por diversidad de especies o por porcentaje de aparición son Fabaceae y Asteraceae. La importancia apícola de estas familias está documentada para la ecorregión del Chaco por Andrada \& Tellería. (2002), Forcone et al., (2003), Cabrera \& Salgado (2006), Salgado (2006); Caccavari \& Fagúndez,
(2010), Salgado et al. (2014) y Aquino et al. (2015). Este hecho se atribuye a su morfología floral y a la oferta de néctar adecuadas al trabajo de pecoreo de las obreras Salgado et al. (2014).

La ecorregión del Chaco fue declarada como Prioridad I (Máxima Prioridad) para los planes de conservación del Banco Mundial y el World Wildlife Foundation (WWF) debido a la notoria degradación que sufrió a lo largo de los años (Dinerstein et al., 1995). El incremento en el desarrollo de trabajos melisopalinológicos de esta región, permite conocer datos sobre los productos melíferos que se generan localmente, brindando una razón más desarrollar políticas de conservación del bosque nativo.

El análisis de las mieles del departamento Graneros (Tucumán), situado en la ecorregión Chaco Seco, contribuye a ampliar el conocimiento sobre los recursos nectaríferos del noroeste del país. Se destacan condiciones muy favorables para la actividad apícola, que se potencia como proveedora de recursos económicos en un área sujeta a recurrentes inundaciones que condicionan el desarrollo de otras actividades agropecuarias.

\section{Conclusiones}

Teniendo en cuenta los resultados obtenidos y la contribución de otros trabajos para la ecorregión del Chaco podemos enunciar las siguientes características para las mieles del departamento Graneros:

- Los tipos polínicos dominantes son Atamisquea emarginata, Prosopis sp. y Sarcomphalus mistol.

- Existe un alto porcentaje de mieles monoflorales (76\%) de las especies antes mencionadas.

- Se refleja alta frecuencia de aparición de polen de especies arbóreas o arbustivas autóctonas.

- Se observó escasa frecuencia de polen de origen exótico. 


\section{Contribución de Autores}

Todos los autores de este trabajo han contribuido por igual a su desarrollo.

\section{Agradecimientos}

Agradecemos a la Fundación Miguel Lillo por brindar financiamiento y permanente apoyo al proyecto. Al Lic. Hugo Ayarde por sus correcciones $\mathrm{y}$ aportes.

\section{Biblografía}

ACCORTI, M., L. PERSANO ODDO, M. PIAZZA \& A. SABATINI. 1986. Schede di caratterizzazione delle principali qualità di miele italiano. Apicoltura 2: 1-35.

ALBARRACÍN, V. N., M. E. GARCÍA \& N. J. F. REYES, 2014. Caracterización y tipificación de mieles de monte del departamento de Graneros provincia de Tucumán. Rep. Argentina, resultados preliminares. Rev. Agr. Agron. Noroeste Argent. 34: 100-103.

ANDRADA, A. C. \& M. C. TELLERÍA. 2002. Botanical origin of honey from south of Calde'n district (Argentina). Grana 41: 58-62.

http://dx.doi.org/10.1080/00173130260045512

AQUINO, D., N. PELLIZSER, D. MIRANDA \& C. SALGADO. 2015. Contenido polínico de mieles de Apis mellifera L. producidas en Misiones, Argentina. Revista Forestal Yvyrareta 22: 8-12.

ARENAS, P. 2003. Etnografia y alimentación entre los toba-ñachilamoleek y Wichi-Ihuku'tas del Chaco Central (Argentina). 1ra ed. Pastor Arenas, Bs. As.

BASILIO, A. \& E. ROMERO. 1996. Contenido polínico de las mieles de la región del Delta del Paraná (Argentina). Darwiniana 34: 113-120.

https://www.jstor.org/stable/23223187

BASILIO, A. M. \& M. NOETINGER. 2002. Análisis polínicos de mieles de la región Chaqueña: Comparación del origen floral entre zonas: Domo central y Esteros, Cañadas y Selvas de Rivera. Rev. Investig. Agropecu. 31: 127-134.

BURGOS, M. \& A. SÁNCHEZ. 2014. Preferencias alimenticias en las mieles inmaduras de Apis mellifera en el Chaco Serrano (Jujuy, Argentina). Bol. Soc. Argent. Bot. 49: 41-50. http://dx.doi.org/10.31055/1851.2372.v49.n1.7820

BURGOS, M. G., A. C. SANCHEZ \& L. C. LUPO. 2015. Análisis polínico de cargas corbiculares de Apis mellifera del Chaco Serrano, Jujuy (Argentina). Lilloa. 52: 3-11. http://lillo.org.ar/publicaciones/lilloa/v52n1/a01
BURKART, R., N. O. BÁRBARO, R. O. SÁNCHEZ \& D. A. GÓMEZ. 1999. Ecorregiones de la Argentina. APN-PRODIA, Buenos Aires.

CABRERA, M. M. 2006. Caracterización polínica de las mieles de la provincia de Formosa, Argentina. Rev. Mus. Argentino Cienc. Nat. 8: 135-142.

CABRERA, M. M. \& C. R. SALGADO. 2006. Contribución al estudio de la flora melífera de la provincia de Formosa, Argentina. Comunicaciones Cientificas y Tecnológicas. Universidad Nacional del Nordeste, Corrientes Argentina. Disponible en http://www.guarani.unne.edu.ar/unnevieja/Web/cyt/ cyt2006/06-Biologia/2006-B-001.pdf. [Acceso: 21 agosto 2019].

CABRERA, M., A. ANDRADA \& L. GALLEZ. 2013. Floración de especies con potencial apícola en el Bosque Nativo Formoseño, Distrito Chaqueño Oriental (Argentina). Bol. Soc. Argent. Bot. 48: 477491. http://digital.cic.gba.gob.ar/handle/11746/6546

CACCAVARI, M. \& G. FAGÚNDEZ. 2010. Pollen spectra of honeys from the Middle Delta of Paraná River (Argentina) and their environmental relationship. Span. J. Agric. Res. 8: 42-52.

http://dx.doi.org/10.5424/sjar/2010081-1142

CARRIZO, E. D. V., M. O. PALACIO, H. J. MÜLLER, M. F. EPSTEIN VITTAR \& F. N. CÉSPEDES. 2015. Especies de interés apícola en la flora del departamento Ojo de Agua, Santiago del Estero, Argentina. Quebracho: Revista de Ciencias Forestales 23: 15-26.

COSTA, M. C., V. A. VERGARA-ROIG \& S. C. KIVATINITZ. 2013. A melissopalynological study of artisanal honey produced in Catamarca (Argentina). Grana 52: 229-237 https://doi.org/10.1080/00173134.2013.819525

COSTA, M. C., M. J. LOYOLA, D. OSÉS, V. A. VERGARA ROIG \& S. C. KIVATINITZ. 2016. Marcadores polínicos en mieles del noroeste de la provincia de Córdoba, Argentina. Darwiniana, nueva serie 4: 277-290.

https://doi.org/10.14522/darwiniana.v4i2.726

DINERSTEIN, E., D. M. OLSON, D. J. GRAHAM, A. L. WEBSTER, S. A. PRIMM, M. P. BOOKBINDER \& G. LEDEC. 1995. Una Evaluación del Estado de Conservación de las Ecoregiones Terrestres de América Latina y el Caribe. WWF- Banco Mundial, Washington D.C. Disponible en: http://documentos. bancomundial.org/curated/es/917091468269687252/ Una-evaluacion-del-estado-de-conservacion-delas-ecoregiones-terrestres-de-America-Latina-y-elCaribe. [Acceso: 21 agosto 2019].

DI RIENZO, J. A., F. CASANOVES, M. G BALZARINI, L. GONZALEZ, M. TABLADA \& C. W. ROBLEDO. 2012. Grupo InfoStat FCA, Universidad Nacional de Córdoba, Argentina. Disponible en: http://www. infostat.com.ar [Acceso: 21 agosto 2019]. 


\section{N. J. F. Reyes et al. - Caracterización de mieles del Chaco tucumano}

DRH. 2017. Dirección de recursos Hídricos Tucumán. Informe Técnico. Documento síntesis. Comisión de emergencia para el tratamiento de la problemática de inundaciones en el sur de la provincia de Tucumán, este de Catamarca y Río Hondo. Disponible en: http://www.recursoshidricos.gov.ar/webdrh/_docs/ CIST-Informe\%20Completo.pdf. [Acceso: 21 agosto 2019].

FAGÚNDEZ, G. A. \& M. A CACCAVARI. 2003. Caracterización polínica y organoléptica de algunas mieles monoflorales del centro de la provincia de Entre Ríos, Argentina. Polen 12: 77-95.

FAGUNDEZ, G. A., P. D. REINOSO \& P. G. ACEÑOLAZA, 2016. Caracterización y fenología de especies de interés apícola en el departamento Diamante (Entre Ríos, Argentina), Bol. Soc. Argent. Bot. 51: 243-267.

FERRÁN, A. M., E. GATTI \& L. A. BALLESTRI. 2002. Análisis de las Fluctuaciones de los Precios de la Miel a Granel. Ciencia Veterinaria 4: 28-34. Disponible en: https/cerac.unlpam.edu.ar/index. php/veterinaria/article/view/1964/1921. [Acceso: 21 agosto 2019].

FLORES, F. F., L. C. LUPO \& N. I. HILGERTH. 2015. Recursos tróficos utilizados por Plebeia intermedia (Apiade, Meliponini) en la localidad de Baritú, Salta, Argentina. Caracterización botánica de sus mieles. Bol. Soc. Argent. Bot. 50: 515-529.

FORCONE,A.\& M.C. TELLERIA. 1998. Caracterización palinológica de las mieles del Valle Inferior del Rio Chubut (Argentina). Darwiniana 36: 81-86.

FORCONE,A.\& M.C. TELLERIA. 2000. Caracterización palinológica de las mieles de la llanura del Rio Senguerr (Chubut, Argentina). Darwiniana 38: 267271.

https://doi.org/10.14522/darwiniana.2014.383-4.172

FORCONE A., O. BRAVO \& M. AYESTARÁN. 2003. Intraannual variations in the pollinic spectrum of honey from the lower valley of the River Chubut (Patagonia, Argentina). Span. J. Agric. Res. 1: 29-36. http://dx.doi.org/10.5424/sjar/2003012-18.

FORCONE, A., G. AYESTARÁN, A. KUTSCHKER \& J. GARCIA. 2005. Palynological characterisation of honeys from the Andean Patagonia (ChubutArgentina) Grana, 44: 202-208.

FORCONE, A. \& A. ANDRADA. 2006. Flora Melifera de las Regiones Pampeana Austral y Patagonia Extra-andina. 1ra ed. EdiUNS, Bahía Blanca.

GARCÍA, M. E., N. J. F. REYES \& H. G. RÍOS. 2012. Atlas Polínico del NOA. Compendio fotográfico de granos de polen de plantas nativas y cultivadas del noroeste argentino. 1ra ed. Editorial Académica Española, Saarbrücken.

GARCÍA, M. E., M. L. ESPECHE \& N. J. F. REYES. 2017. Palinoteca del Laboratorio de Palinología de Fundación Miguel Lillo (PAL-TUC), Tucumán,
Argentina. Boletín de la Asociación Latinoamericana de Paleobotánica y Palinología 17: 1-11. Disponible en: http:/www.palino.com.ar/alpp/BoletinesALPP/ ALPP-Boletin-vol17.pdf. [Acceso: 21 agosto 2019].

GONZÁLES, J. A., H. H. SALAS \& J. M. CHANI. 2009. Reserva natural de uso múltiple "Chaco Tucumano" (Tucumán, Argentina). Integrando la preservación ambiental con el desarrollo rural en el sudeste de la provincia de Tucumán. Serie Conservación de la naturaleza Fundación Miguel Lillo 18:1-18.

HESSE, M., H. HALBRITTER, R. ZETTER, M. WEBER, R BUCHNE, A. FROSCH-RADIVO \& S. ULRICH. 2009. Pollen terminology: An ilustrated handbook. Springer, Viena.

JATO, M., M. IGLESIAS \& V. RODRIGUEZ-GRACIA. 1994. A contribution to the environmental relationship of the pollen spectra of honeys from Orense (NW Spain). Grana 33: 260-267. https://doi.org/10.1080/00173139409429008

JEAN-PROST, P. 1989. Apicultura. Ediciones MundiPrensa, Madrid.

LOUVEAUX, J., A. MAURIZIO \& G. VORWHOL. 1978. Methods of Melissopalynology. Bee World 59: 139-157. https://doi.org/10.1080/0005772X.1978.11097714

LUSARDI, M., D. PRADO \& S. GATUSSO. 2005. Contenido polínico de las mieles del sur de la Provincia de Santa Fe (Argentina). Bol. Soc. Argent. Bot. 40: 85-90.

MAGAÑA-MAGAÑA, M. A., J. R. SANGINÉSGARCÍA, P. E. LARA Y LARA, L. SALAZARBARRIENTOS \& C. E. LEYVA-MORALES. 2017. Competitividad y participación de la miel mexicana en el mercado mundial. Rev. Mex. Cienc. Pecu. 8: 4352. http://dx.doi.org/10.22319/rmcp.v8i1.4304

MARKGRAF, V. \& H. D'ANTONI. 1978. Pollen Flora of Argentina. Modern Spore and Pollen Types of Pteridophyta, Gymnospermae and Angiospermae. 1ra ed. The University of Arizona Press, Arizona.

MENDEZ, M. V., A. C. SANCHEZ, F. F. FLORES \& L. C. LUPO. 2016. Análisis polínico de mieles inmaduras en el sector oeste de las yungas de Jujuy (Argentina). Bol. Soc. Argent. Bot. 51: 449-462. http://dx.doi.org/10.31055/1851.2372.v51.n3.15390

MINISTERIO DE AGROINDUSTRIA DE LA NACIÓN. 2018. Cadena Apícola. Informe de coyuntura mensual. Septiembre 2018. Disponible en: http:// www.alimentosargentinos.gob.ar/HomeAlimentos/ Apicultura/documentos/informedecoyunturaENERO. pdf. [Acceso 1 noviembre 2018].

MONTENEGRO, G., R. PIZARRO, G. ÁVILA, R. CASTRO, C. RÍOS, O. MUÑOZ, F. BAS \& M. GÓMEZ. 2003. Botanical origin and chemical properties of honeys from an Arid Mediterranean Region of Chile Cienc. Investig. Agrar. 30(3): 161174. http://dx.doi.org/10.7764/rcia.v30i3.1311 
MONTENEGRO, S. B., \& C. CHIFA. 2004. Estudio integral de mieles del Chaco. Comunicaciones Científicas y Tecnológicas. Universidad Nacional del Nordeste, Corrientes Argentina. Disponible en http://www.guarani.unne.edu.ar/unnevieja/Web/cyt/ cyt/2002/07-Tecnologicas/T-050.pdf. [Acceso: 21 agosto 2019].

MORELLO, J., A. RODRIGUEZ \& M. SILVA. 2012. Ecorregión del Chaco Seco. En: GEPAMA, FADU, UBA (eds.) Ecorregiones y Complejos Ecosistémicos Argentinos, pp. 800 GEPAMA, FADU, UBA, Buenos Aires.

NAAB, O. \& M. A. TAMAME. 2007. Flora Apícola en la región del Monte de la provincia de La Pampa, Argentina. Bol. Soc. Argent. Bot. 42: 251-259.

NUÑEZ CAMELINO, A. A. 2000. Determinación del potencial apícola de las secciones $1^{\circ}, 2^{\circ}$ y $4^{\circ}$ del Departamento Saladas de la Provincia de Corrientes. Comunicaciones Científicas y Tecnológicas. Universidad Nacional del Nordeste, Corrientes, Argentina. Disponible en http://wwwl.unne.edu.ar/ unnevieja/Web/cyt/cyt/2000/5_agrarias/a_pdf/a_079. pdf. [Acceso: 21 agosto 2019].

PAILHE, L. E \& E. R. POPOLIZIO. 1971. Estudio Analítico de mieles tucumanas. Fac. de Agron. y Zoot. UNT Miscelánea 38: 1-5.

PHILIPPE, J. M. 1990. Guía del Apicultor. Mundi-Prensa, Madrid.

PIRE, S. M., L. M. ANZÓTEGUI \& G. A. CUADRADO. 1998. Flora Polínica del Nordeste Argentino, Vol. 1. 1ra ed. EUDENE-UNNE, Corrientes.

PIRE, S. M., L. M. ANZÓTEGUI \& G. A. CUADRADO. 2001. Flora Polínica del Nordeste Argentino, Vol. 2. 1ra ed. EUDENE-UNNE, Corrientes.

PIRE, S. M., L. M. ANZÓTEGUI \& G. A. CUADRADO. 2006. Flora Polínica del Nordeste Argentino, Vol. 3. 1ra ed. EUDENE-UNNE, Corrientes.

POPOLIZIO, E. R. \& L. A. PAILHÉ. 1973. Espectro Floral Apícola - XXIV Congreso Internacional de Apicultura - Apimondia - Bs. As. Argentina.

PORTAL APÍCOLA. 2015. Colmenas bajo agua en Tucumán. Disponible en: http://api-cultura.com/ colmenas-bajo-agua-en-tucuman/. [Acceso 5 noviembre 2018]

QUIROGA, V., G. LUNA \& S. MARTÍNEZ. 2008. Origen Botánico y Propiedades Físicas y Químicas de Mieles Primaverales de Cinco Localidades del Valle Central de Catamarca. Ciencia. 3(5): 85. Disponible en http://www.exactas.unca.edu.ar/revista/v50/pdf/ RevCiencia5-5.pdf. [Acceso: 21 agosto 2019].

RESOLUCIÓN SAGPYA N 1051/94. 1994. Tipificación por origen botánico. Secretaria de Agricultura, Ganadería,PescayAlimentación.Disponibleen:https:// apicultura.fandom.com/wiki/Resoluci\%C3\%B3n SAGPyA_N\%C2\%BA_1051/94. [Acceso: 21 agosto 2019].
RESOLUCIÓN SAGPYA Nº 274/95. 1995. Tipificación por origen botánico. Modificatoria de Resolución 1051/94. Secretaria de Agricultura, Ganadería, Pesca y Alimentación. Disponible en: https:// apicultura.fandom.com/wiki/Resoluci\%C3\%B3n SAGPyA_N\%C2\%BA_1051/94. [Acceso: $2 \overline{1}$ agosto 2019].

SÁENZ-DE RIVAS, C. 1978. Polen y esporas (Introducción a la Palinología y vocabulario palinológico). 1 ra ed. Blume Ediciones, Madrid.

SALGADO, C. R. \& S. M PIRE. 1998. Análisis polínico de mieles del Noroeste de la provincia de Corrientes (Argentina). Darwiniana 36: 87-93.

SALGADO, C. R. \& S. PIRE. 1999. Contribución al conocimiento del contenido polínico de mieles de Corrientes. En M. GRIFFIN (ed.). X Simposio Argentino de Paleobotánica y Palinología, pp 9599. Asociación Paleontológica Argentina, Buenos Aires.

SALGADO, C. R. 2006. Flora melifera en la provincia del Chaco. PROSAP-Ministerio de la Producción del Chaco, Chaco.

SALGADO, C. R., G. PIEZKO \& M. C. TELLERIA. 2014. Aporte de la melisopalinología al conocimiento de la flora melífera de un sector de la provincia fitogeográfica chaqueña, Argentina. Bol. Soc. Argent. Bot. 49: 513-524.

SALGADO, C. R. 2016. Caracterización botánica y geográfica de las mieles producidas por Apis mellifera L. en la provincia del Chaco, a partir de su composición polínica y parámetros físicoquímicos. Tesis Doctoral, UNNE, Corrientes.

SÁNCHEZ, A. C. \& L. LUPO. 2009. Asteraceae de Interés en la Melisopalinología. Bosque Montano de las Yungas (Jujuy-Argentina). Bol. Soc. Argent. Bot. 44: 57-64.

SÁNCHEZ, A. C. \& N. D. VIGNALE. 2009. Flora Apícola de la Quebrada de Humahuaca. Arnaldoa 16: 101-108.

SANCHEZ, A. \& L. LUPO. 2011. Origen botánico y geográfico de las mieles de El Fuerte, Departamento de Santa Bárbara, Jujuy, Argentina. Bol. Soc. Argent. Bot. 46: 105-111.

SÁNCHEZ, A. C. \& L. C. LUPO. 2016. Caracterización Palinológica de Mieles de la Zona I: Prepuna. (Jujuy, Argentina). Bol. Soc. Argent. Bot. 51: 441-448.

SODERSTRON, T. R. \& C. E. CALDERON. 1971. Insect pollination in tropical rain forest grasses. Biotropica 3: 1-16. http://dx.doi. org/10.2307/2989701

TELLERÍA, M. C. 1992. Caracterización botánica y geográfica de las mieles de la provincia Fitogeográfica Pampeana (República Argentina) I: Distrito Oriental. Darwiniana 31: 345-350.

TELLERÍA, M. C. 1995. Plantas de importancia apícola del distrito Oriental de la región 


\section{N. J. F. Reyes et al. - Caracterización de mieles del Chaco tucumano}

Pampeana (Argentina). Bol. Soc. Argent. Bot. 30: 131-136.

TELLERÍA, M. C. 1996. Caracterización botánica y geográfica de las mieles de la provincia Fitogeográfica Pampeana (República Argentina) III: Noreste de la Provincia de La Pampa. Darwiniana 34: 245-249.

TELLERÍA, M. C. 2001. El polen de las mieles, un indicador de su procedencia botánica y geográfica. Ciencia Hoy 11(62): 63-66.
TORRELLA, S. A. \& J. ADAMOLI. 2006. Situación ambiental de la ecorregión del Chaco Seco. En: A. BROWN, U. MARTINEZ ORTIZ, M. ACERBI, \& J. CORCUERA (eds.), La Situación Ambiental Argentina 2005, pp. 73-100. Fundación Vida Silvestre Argentina, Buenos Aires.

ZUCCARDI, R. B. \& G. S. FADDA. 1985. Bosquejo agrológico de la provincia de Tucumán. Fac. de Agron. Y Zoot. UNT Miscelánea 86: 1-5. 
